\title{
Consumo de oxígeno, crecimiento y utilización del alimento durante el desarrollo larval del besugo Pagrus pagrus (Linné, 1758) (Pisces: Sparidae)
}

\author{
Oxygen consumption, growth and food utilization during the larval development of \\ red porgy Pagrus pagrus (Linné 1758) (Pisces: Sparidae)
}

\author{
Eddie O. Aristizabal ${ }^{1}$ \\ ${ }^{1}$ Instituto Nacional de Investigación y Desarrollo Pesquero (INIDEP), \\ Paseo V. Ocampo \# 1, Escollera Norte, B7602HSA Mar del Plata, Argentina \\ eddie@inidep.edu.ar
}

\begin{abstract}
Knowledge of the life history of fish larvae is of practical importance to understand variations in the survival rate not only for cultivated fish but also for fishery biology studies. With the aim of recognizing critical changes during the first days of life of red porgy larvae, the oxygen consumption, growth, and food utilization were studied. After hatching, the larvae were transferred to two $400 \mathrm{~L}$ fiberglass tanks with microalgae (Nannochloropsis oculata) and enriched rotifers (Brachionus plicatilis) as initial food. During the incubation and larval rearing, water temperature was kept at $18.0 \pm 0.5^{\circ} \mathrm{C}$ and salinity ranged from 33.0 to $34.0 \mathrm{psu}$.

Results indicate a decrease in larval growth between day 3 and 4 after hatching, when larvae opened their mouths, began exogenous feeding and have almost exhausted their yolk sac and oil globule. At the end of this period, variations in the carbon and nitrogen content were also detected. Oxygen consumption showed an isometric mass-specific change with an inflexion at day 6 after hatching, which was coincident with the inflation of the gas bladder and the development of gills. The nitrogen coefficient (NQ) revealed a sudden change in the utilization of amino acids and lipids from endogenous reserves between the embryo and the newly hatched larvae. Hatching and first feeding time are two critical changing moments for the larval survival of this species.
\end{abstract}

Key words: Oxygen, metabolism, fish
Resumen.- El estudio del desarrollo larval en peces es importante para comprender las variaciones en la supervivencia observadas tanto a nivel de cultivo como en los estudios biológico-pesqueros. Por ello, se registró el consumo de oxígeno, el crecimiento y la utilización del alimento en larvas de $P$. pagrus criadas en cautiverio, con el objeto de recabar información acerca de los cambios críticos que ocurren durante los primeros días de vida de esta especie. Luego de la eclosión, las larvas se sembraron en dos tanques de 400 L con microalgas (Nannochloropsis oculata) y rotíferos (Brachionus plicatilis) enriquecidos como alimento. La incubación y cría de larvas se realizó a $18,0 \pm 0,5^{\circ} \mathrm{C}$ y la salinidad se mantuvo entre 33,0 y 34,0 ups.

Los resultados indican una desaceleración del crecimiento larval entre los 3 y 4 días después de la eclosión, coincidiendo con la apertura de la boca, el inicio de la alimentación externa y el agotamiento del saco vitelino y de la gota oleosa. Al término de este período también se detectaron variaciones en el contenido de carbono y nitrógeno. El consumo de oxígeno mostró un cambio isométrico en relación al peso, con un punto de inflexión al día 6 después de la eclosión, coincidiendo con la aparición de la vejiga natatoria y el desarrollo de las branquias. El coeficiente de nitrógeno (NQ) reveló un cambio en la utilización de los aminoácidos y lípidos de las reservas internas entre el embrión y la larva recién eclosionada. Tanto la eclosión como el inicio de la alimentación son dos momentos de cambios críticos en la supervivencia larval de esta especie.

Palabras clave: Oxígeno, metabolismo, peces 


\section{Introducción}

El besugo Pagrus pagrus (Linné) es una especie de amplia distribución en el Mediterráneo y en las costas oriental y occidental del Océano Atlántico (Manooch 1976). Dentro del Atlántico sudoccidental se encuentra naturalmente distribuido a lo largo de las costas de la Provincia de Buenos Aires, Argentina, entre los 10 y 50 $\mathrm{m}$ de profundidad (Cotrina 1989). Es una especie demersal que muestra a lo largo de la costa bonaerense una preferencia por los fondos rocosos, conformando un importante recurso pesquero. Las capturas de esta especie llegaron a un máximo de $15.300 \mathrm{t}_{\text {año }}{ }^{-1}$ en la década de 1980, para posteriormente decrecer hasta ubicarse en $2.200 \mathrm{t} \mathrm{año}^{-1}$ en 2004 (SAGPyA 2004). Esta situación, junto al alto valor comercial de esta especie en el área del Mediterráneo y el sur de España (Hernández-Cruz et al. 1999), han hecho que su cultivo haya recibido considerable atención en los últimos años (Aristizabal et al. 1997a, b, Hernández-Cruz et al. 1999, Mihelakakis et al. 2001, Mylonas et al. 2004, Radonic et al. 2005).

El estudio del desarrollo larval de los peces es de capital importancia para comprender los factores que operan durante los primeros estadios de vida, tanto para ayudar a mejorar las técnicas de acuicultura (Ishibashi et al. 1992, Phil et al. 1992) como para brindar información útil aplicable a la explotación y manejo de la pesquería (Tanaka 1985, Wu 2002). Durante la producción de juveniles de $P$. pagrus, es normal que se presenten fluctuaciones en las tasas de supervivencia larval (Aristizabal en prensa, Radonic et al. 2005), las cuales necesitan ser estudiadas. El objetivo del presente trabajo es conocer los cambios fisiológicos que ocurren durante los primeros días de vida de esta especie a través de la medición de los parámetros energéticos.

\section{Materiales y métodos}

Las larvas utilizadas en este estudio provienen de la puesta espontánea de los reproductores de $P$. pagrus pertenecientes al Proyecto de Cultivo de Besugo del INIDEP. Inmediatamente después de la puesta, los huevos se recogieron en una red planctónica de $300 \mu \mathrm{m}$ de malla y se transfirieron a un tanque circular de fibra de vidrio de 400 L para su incubación. Las larvas recién eclosionadas se utilizaron para sembrar dos tanques de $400 \mathrm{~L}$ con 26.000 larvas cada uno. Los tanques se ubicaron dentro de una sala climatizada con recirculación cerrada de agua de mar. La concentración de microalgas (Nannochloropsis oculata) en el tanque de larvas se mantuvo entre $0,5-1,0 \times 10^{6}$ cél $\mathrm{mL}^{-1}$ durante todo el experimento. La incubación y cría de larvas se realizó a $18,0 \pm 0,5^{\circ} \mathrm{C}$ de temperatura. $\mathrm{La}$ salinidad se mantuvo entre 33,0 y 34,0 ups. La alimentación inicial de las larvas estuvo compuesta por rotíferos (Brachionus plicatilis) enriquecidos con ácidos grasos y vitaminas (DHA-Ce, Oriental Ind., Japan). La densidad inicial de rotíferos en el tanque se fijó en 3 rot. $\mathrm{mL}^{-1}$ al día 3 después de la eclosión (DE), hasta llegar paulatinamente a los 10 rot. $\mathrm{mL}^{-1}$ al día 7 DE.

Las observaciones morfométricas se realizaron tomando muestras a intervalos de $24 \mathrm{~h}$ hasta las $168 \mathrm{~h}$ DE. En cada intervalo se recolectaron 50 larvas de cada tanque. Se registraron la longitud total (Lt), el tamaño del saco vitelino y el diámetro de la gota oleosa con un microscopio estereoscópico Nikon modelo SE con ocular micrométrico Zeiss KF 10x/18. Todas las observaciones se realizaron en individuos vivos (Klaoudatos et al. 1990) anestesiados con una gota de una solución de benzocaína : acetona $(2: 1 \mathrm{p} / \mathrm{v})$. El volumen del saco vitelino (Vsv) y de la gota oleosa (Vgo) se calcularon usando las fórmulas propuestas por Cetta \& Capuzzo (1982), quienes consideran al saco vitelino como una elipse y a la gota oleosa como una esfera:

$$
\begin{aligned}
& V s V=\pi / 6 L H^{2} \\
& V g o=4 / 3 \pi r^{3}
\end{aligned}
$$

donde, $\mathrm{L}$ es el eje mayor de la elipse; $\mathrm{H}$ es el eje menor (vertical) de la elipse y $\mathrm{r}$ el radio de la esfera en $\mathrm{mm}$. La disminución del volumen de la gota oleosa y saco vitelino se ajustaron a una curva exponencial del tipo:

$$
V=V_{o} e^{-s t}
$$

donde $\mathrm{V}$ es el volumen $\left(\mathrm{mm}^{3}\right), \mathrm{V}_{\mathrm{o}}$ es el volumen al momento de la eclosión $\left(\mathrm{mm}^{3}\right)$, s es la tasa específica de consumo $\mathrm{h}^{-1} \mathrm{y}$ t son los días después de la eclosión.

Los valores de tasa de crecimiento $\mathrm{g}$ (incremento medio de la talla $\mathrm{h}^{-1}$ ) y de tasa de utilización del saco vitelino u (disminución media del volumen del saco vitelino $\mathrm{h}^{-1}$ ) se obtuvieron de acuerdo a las siguientes ecuaciones: 


$$
\begin{gathered}
g=\frac{\left(L \arg o_{i}-L \arg o_{i-1}\right)}{t \frac{\left(L \arg o_{i}-L \arg o_{i-1}\right)}{2}} \times 100 \\
u=\frac{\left(V s v_{i}-V s V_{i-1}\right)}{t \frac{\left(L \arg o_{i}-L \arg o_{i-1}\right)}{2}} \times 100
\end{gathered}
$$

donde i se refiere a la medición al final del período i, i-l es la medición al principio del período $\mathrm{i}, \mathrm{y} \mathrm{t}$ es el tiempo transcurrido entre mediciones sucesivas (24 h). La eficiencia del desarrollo (Ed) se calculó de acuerdo a la relación $\mathrm{u} / \mathrm{g}$, propuesta por Klaoudatos et al. (1990). Se realizó la prueba de ANOVA sobre significancia de medias.

La relación entre la tasa de crecimiento y el tiempo después de la eclosión de las larvas de besugo se calculó utilizando las ecuaciones de von Bertalanffy y Logística (Ricker 1979):

von Bertalanffy: $L=L_{\text {inf }}\left[1-e^{-b(t+c)}\right]$

Logística $\quad L=\frac{a}{1+b e^{-c t}}$

donde $\mathrm{L}$ es la estimación de la longitud a un intervalo dado, $\mathrm{L}_{\text {inf }}$ es la talla máxima alcanzada durante el período estudiado (mm), y $a, b$ y $c$ son los coeficientes calculados utilizando los datos de talla a intervalos de tiempo.

Para los análisis químicos de las larvas se tomaron muestras con un tubo plástico de PVC en diferentes lugares a lo largo de la columna de agua de los tanques de cría. La muestra total se pasó por papel de filtro para recoger las larvas que posteriormente se lavaron con agua destilada. El peso seco (PS) se determinó secando muestras de 50 larvas en una estufa a $90^{\circ} \mathrm{C}$ hasta peso constante. El contenido de carbono (C) se estimó por el método de Gaudette et al. (1974) modificado por ElRayis (1985), mientras que el nitrógeno $\left(\mathrm{N}_{2}\right)$ se determinó por el método micro-Kjeldahl (factor de conversión 6,25 $\mathrm{x} \mathrm{N}_{2}$ ). La proporción de lípidos se estimó por el método de Twisselmann (Antonacopoulos 1968). Brevemente, el método consiste en la extracción de lípidos de la muestra seca y pulverizada utilizando éter de petróleo, libre de peróxidos, hasta su agotamiento y posterior evaporación del medio de extracción, secado del extracto y pesado luego de enfriado. El contenido de ceniza se determinó por calcinación a $600^{\circ} \mathrm{C}$ por $6 \mathrm{~h}$ y posterior pesada a 0,001 g. Las muestras se analizaron por triplicado. La energía total se calculó en base a los factores de conversión del Programa Biológico Internacional (Kamler 1992), que son 23,0 $\mathrm{J} \mathrm{mg}^{-1}$ para proteínas, 39,8 $\mathrm{J} \mathrm{mg}^{-1}$ para lípidos y $17,2 \mathrm{~J} \mathrm{mg}^{-1}$ para carbohidratos.

El consumo de oxígeno $\left(\mathrm{O}_{2}\right)$ y la excreción de amonio $\left(\mathrm{NH}_{4}^{+}\right)$se midieron en un respirómetro cerrado a $18,0 \pm 0,5^{\circ} \mathrm{C}$ de temperatura, conectado a un equipo ORION 720A equipado con electrodos Cole-Parmer para $\mathrm{O}_{2}$ disuelto y $\mathrm{NH}_{4}^{+}$. Cada ensayo se realizó en grupos de 100 huevos o 50 larvas por un tiempo promedio de $4 \mathrm{~h}$, luego de un periodo de $3 \mathrm{~h}$ de ayuno en los casos en que correspondiera. El respirómetro consistió en frascos de vidrio calibrados y cerrados con polietileno de $2 \mathrm{~mm}$ de espesor y encintados en todas las uniones para asegurar el hermetismo. Se tomó especial precaución en eliminar todo el aire y las burbujas existentes entre el nivel de agua y el polietileno. Los frascos se rotaron a mano suavemente a intervalos regulares y antes de las mediciones. Los huevos y larvas utilizados fueron cuidadosamente seleccionados en busca de anormalidades morfológicas. Cada medición se realizó por triplicado con un blanco sin huevos ni larvas. El consumo de oxígeno $\mathrm{VO}_{2}$, expresado como nmol larva ${ }^{-1} \mathrm{~h}^{-1}$, se calculó en base a la ecuación de Cech (1990):

$$
V O_{2}=\frac{\Delta p O_{2} x V H_{2} O}{N x \Delta t}
$$

donde $\Delta p \mathrm{O}_{2}$ es la diferencia en las lecturas de tensión de oxígeno del respirómetro entre el blanco y los grupos experimentales al final de cada período, $\mathrm{VH}_{2} \mathrm{O}$ es el volumen de agua (L) del respirómetro, $\mathrm{N}$ es el número de huevos o larvas, $\mathrm{y} \Delta t$ es el tiempo transcurrido (h) en el respirómetro. El factor de conversión de oxígeno utilizado fue $13,84 \mathrm{~J} \mathrm{mgO}_{2}{ }^{-1}$ (Guinea \& Fernández 1997). La tasa de consumo de oxígeno obtenida en el presente trabajo es la correspondiente al metabolismo de rutina, la cual mide el metabolismo basal más los gastos involucrados en el mantenimiento del equilibrio y natación espontánea. Para los cálculos catabólicos se tomó el coeficiente respiratorio (RQ) para peces carnívoros $(0,8)$.

Se efectuó un análisis de regresión lineal entre el consumo de oxígeno y el peso de las larvas. La prueba de isometría sobre la pendiente $b$ de la regresión se realizó utilizando el estadístico: 


$$
T_{o b s}=\frac{|b-1|}{S b}
$$

La excreción de amonio $\left(\mathrm{VNH}_{4}^{+}\right.$, expresada en nmol larva ${ }^{-1} \mathrm{~h}^{-1}$ ) se calculó en base a:

$$
\mathrm{VNH}_{3}=\frac{\Delta\left[\mathrm{NH}_{4}^{+}\right]_{x} \mathrm{VH}_{2} \mathrm{O}}{\mathrm{N} x \Delta t}
$$

donde $\Delta\left[\mathrm{NH}_{4}^{+}\right]$es la diferencia en la concentración total de amonio del agua entre el blanco y los grupos experimentales.

\section{Resultados}

Los huevos de besugo son esféricos, con un diámetro medio de $903 \pm 12 \mu \mathrm{m}$. Contienen una única gota oleosa que varía entre $180-220 \mu \mathrm{m}$ de diámetro. La eclosión ocurrió a las $50 \mathrm{~h}$, con una tasa del $87 \%$. Las larvas recién eclosionadas de besugo presentaron un largo total medio (Lt) de 2,32 $\pm 0,12 \mathrm{~mm}$ (Tabla 1).

\section{Tabla 1}

Tasa de crecimiento (g) (\% del Lt/día) ( \pm DE), tasa de utilización del saco vitelino (u) (\% del Vsv/día) y eficiencia de desarrollo $(\mathrm{Ed}=\mathrm{u} / \mathrm{g})\left(\mathrm{mm}^{3}\right)$ estimados para larvas de besugo (n $=2$ x 50, $P<0,01)$ a $18^{\circ} \mathrm{C}$

Growth rate $(\mathrm{g})(\% \mathrm{Lt} /$ day $)( \pm \mathrm{SD})$, yolk sac utilization rate (u) $(\% \mathrm{Vsv} /$ day $)$, and efficiency of development $(\mathrm{Ed}=\mathrm{u} / \mathrm{g})$ $\left(\mathrm{mm}^{3}\right)$ for red porgy larvae $(\mathrm{n}=2 \times 50, P<0,01)$ at $18^{\circ} \mathrm{C}$

\begin{tabular}{ccccc}
\hline $\begin{array}{c}\text { TDE } \\
(\text { días })\end{array}$ & $\begin{array}{c}\mathrm{Lt} \pm \mathrm{DE} \\
(\mathrm{mm})\end{array}$ & $\mathrm{g}$ & $\mathrm{u}$ & $\mathrm{Ed}$ \\
\hline 0 & $2,32 \pm 0,12$ & - & - & - \\
1 & $2,86 \pm 0,12$ & 0,8810 & 0,3515 & 0,3989 \\
2 & $3,11 \pm 0,10$ & 0,3446 & 0,0532 & 0,1543 \\
3 & $3,24 \pm 0,07$ & 0,1706 & 0,0207 & 0,1212 \\
4 & $3,32 \pm 0,15$ & 0,0966 & 0,0051 & 0,0527 \\
5 & $3,54 \pm 0,13$ & 0,2723 & & \\
6 & $3,75 \pm 0,12$ & 0,2401 & & \\
7 & $4,16 \pm 0,25$ & 0,4319 & & \\
\hline
\end{tabular}

TDE: tiempo después de la eclosión; Lt: largo total; DE: desviación estándar
La ganancia promedio en largo total $(\mathrm{Lt})$ de las larvas osciló entre $0,076 \mathrm{~mm} \mathrm{~d}^{-1}$ y $0,547 \mathrm{~mm} \mathrm{~d}^{-1}$ durante el período estudiado, mientras que la ganancia máxima se observó en el estadio de 1 día DE $(0,023$ $\mathrm{mm} \mathrm{h}^{-1}$ ) (Tabla 1). La tasa de crecimiento fue constante durante los primeros 7 días DE, excepto por una inflexión entre los días 3 y $4 \mathrm{DE}$ cuando el crecimiento parece detenerse momentáneamente, coincidiendo con la apertura de la boca y el inicio de la alimentación exógena (Fig. 1). De aquí se desprende un patrón de crecimiento de tres fases: a) rápido crecimiento hasta el punto de inflexión, b) crecimiento lento entre los 3 y 4 días $\mathrm{DE}, \mathrm{y}$ c) una tercer fase nuevamente de rápido crecimiento.

Los cálculos de los parámetros de la curva de crecimiento estándar dieron las siguientes ecuaciones de crecimiento ( $t$ entre 1 y 7 días después de la eclosión):

$$
\text { von Bertalanffy } \begin{aligned}
L & =4,41389\left[1-e^{-0,17243(t+0,93113)}\right] \\
r^{2} & =0,974
\end{aligned}
$$

Logística

$$
\begin{aligned}
L & =\frac{4,20805}{1+0,56745 e^{-0,27669 t}} \\
\mathrm{r}^{2} & =0,978
\end{aligned}
$$

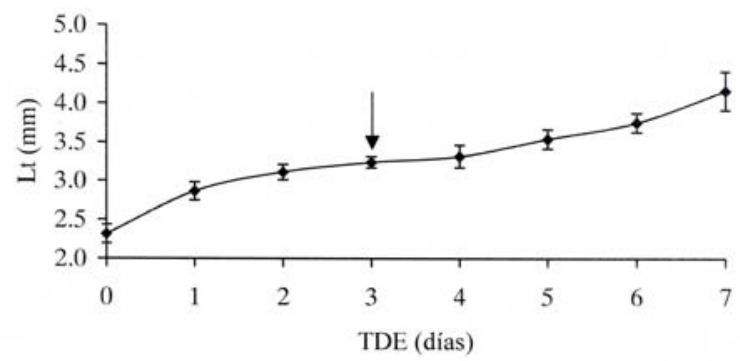

Figura 1

Relación entre el largo total (Lt) de la larva de besugo y el tiempo después de la eclosión (TDE). Las barras verticales representan la desviación estándar. La flecha indica el momento de la apertura de la boca

Relationship between total length (Lt) and time after hatching (TDE) of red porgy larvae. Vertical bars indicate standard deviation. The arrow indicates the time of mouth opening 
El consumo del saco vitelino se inició casi inmediatamente después de la eclosión, mientras que el consumo de la gota oleosa comenzó cuando el saco vitelino estaba en avanzado estado de absorción (Fig. 2). Las ecuaciones que describen el cambio de los volúmenes $\left(\mathrm{mm}^{3}\right)$ del saco vitelino (Vsv) y la gota oleosa (Vgo) en el tiempo $(\mathrm{t})$, son $V_{s v}=V_{o} e^{-1,51009 t}\left(\mathrm{r}^{2}=\right.$ $0,9943)$ y $V_{g o}=V_{o} e^{-0,22591 t}\left(\mathrm{r}^{2}=0,9987\right)$.
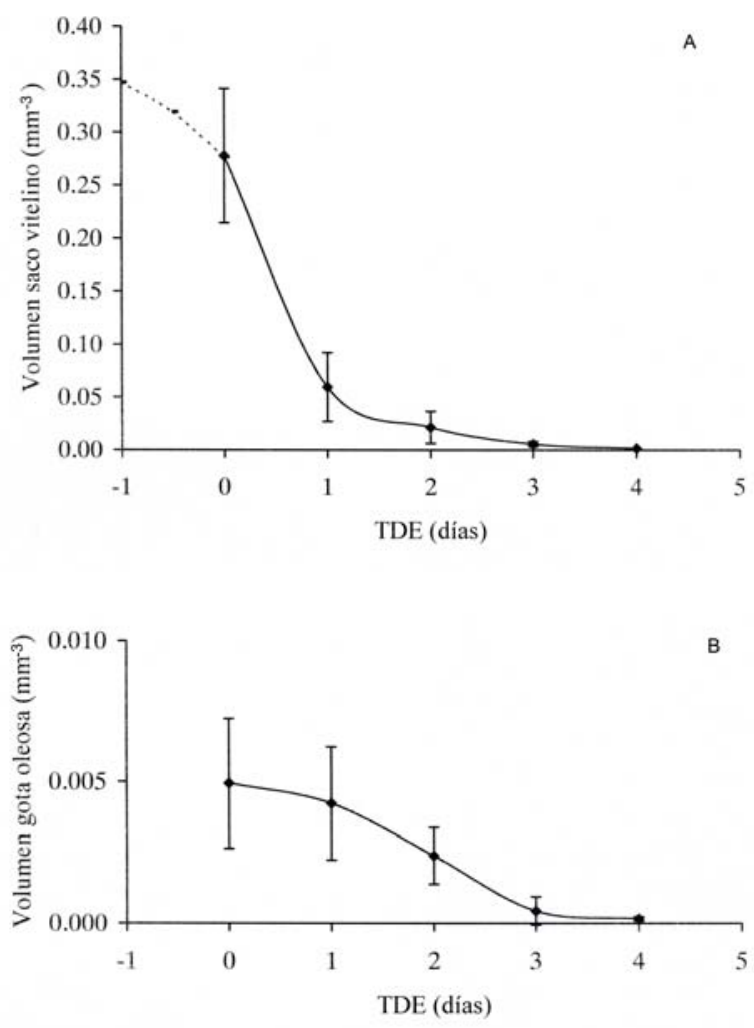

Figura 2

Volumen del saco vitelino (A) y la gota oleosa (B) en larvas de besugo. La línea punteada indica los valores de pre-eclosión (PE) teóricos en base al consumo típico del vitelo en peces teleósteos (Heming \& Buddington 1988). Las barras verticales representan la desviación estándar

Yolk-sac (A) and oil drop (B) volume of red porgy larvae. Dot line indicates theoretical values before hatching (PE) based on the classical yolk consumption in teleost fishes (Heming \& Buddington 1988). Vertical bars represent standard deviation
La eficiencia del desarrollo (Ed) se calculó hasta el día en que el saco vitelino y la gota oleosa fueron totalmente reabsorbidos (Fig. 3). Las primeras larvas con alimento en el tracto digestivo se detectaron a los 4 días DE, existiendo un período de aproximadamente 24 $\mathrm{h}$ entre la apertura de la boca y la aparición de alimento en el intestino.

El PS libre de cenizas de las larvas recién eclosionadas estuvo en promedio en $27,5 \mu \mathrm{g}$ (Tabla 2), el contenido de cenizas en $11,67 \%$ y el contenido calórico en $661,34 \mathrm{~mJ}$ larva $^{-1}$. Durante el estadio vitelino, el contenido de carbono (C) decreció levemente desde la eclosión, mientras que el nitrógeno $\left(\mathrm{N}_{2}\right)$ se mantuvo constante. La relación $\mathrm{C} / \mathrm{N}$, consecuentemente, también decreció pero no lo hizo en forma significativa (Tabla 2). El PS también decreció levemente hasta el día 4 (Tabla 2), siguiendo un patrón similar al C. El contenido de energía de los principales substratos mostró el mismo patrón que el contenido de C, al cual está directamente relacionado (Fig. 4). Al acabarse el vitelo, las larvas tenían un $64 \%$ de su contenido de energía al momento de eclosionar. Los cambios en el contenido de ceniza desde la eclosión fueron más erráticos y variables. A partir del día $6 \mathrm{DE}$, el contenido de $\mathrm{C}, \mathrm{N}_{2}$ y el PS comenzaron a incrementarse (Tabla 2). La tasa de excreción de amonio al momento de la eclosión estuvo en $0,8 \mathrm{nmol}$ larva $^{-1} \mathrm{~h}^{-1}$, siendo baja en las primeras horas de vida de las larvas, para luego incrementarse gradualmente (Fig. $5)$. No se registraron diferencias significativas entre los individuos de los tanques experimentales $(P<0,01)$.

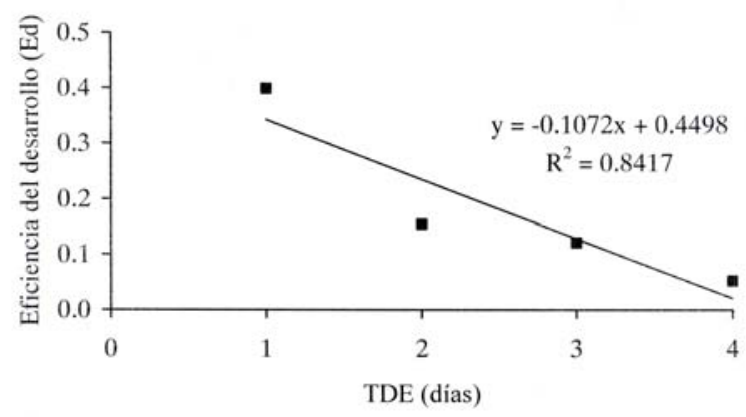

Figura 3

Relación entre la eficiencia del desarrollo larval y el tiempo después de la eclosión (TDE) en larvas de besugo

Relationship between the larval development efficiency and the time after hatching (TDE) in red porgy larvae 
Tabla 2

Peso seco (PS), contenido de carbono (C), nitrógeno $\left(\mathrm{N}_{2}\right)$, ceniza, energía $(\mathrm{J} / \mathrm{mg})$ y relación $\mathrm{C} / \mathrm{N}$ en larvas de besugo $(\mathrm{n}=3 \mathrm{x}$ $150, P<0,01)$. Todos los valores están expresados en biomasa seca de materia orgánica (MO) libre de cenizas \pm DE

Dry weight (PS), carbon $(\mathrm{C})$, nitrogen $\left(\mathrm{N}_{2}\right)$, ash and energy $(\mathrm{J} / \mathrm{mg})$ contents and the relationship of $\mathrm{C} / \mathrm{N}$ in red porgy larvae $(\mathrm{n}=3 \mathrm{x}$ $150, \mathrm{P}<0.01)$. Values are expressed in dry weight of organic matter $(\mathrm{MO})$ ash free $\pm \mathrm{SD}$

\begin{tabular}{cccccccc}
\hline & & \multicolumn{5}{c}{ Cenizas } & Energía \\
Día & PS $(\mu \mathrm{g})$ & $\% \mathrm{C}$ & \%NB $_{2 \mathrm{~B}}$ & $(\%)$ & Energía $(\mathrm{J} / \mathrm{mg})$ & $(\mathrm{mJ} / \mathrm{ind})$ & $\mathrm{C} / \mathrm{N}$ \\
\hline 0 & $27,5 \pm 4,6$ & $55,7 \pm 1,6$ & $10,5 \pm 0,8$ & 11,7 & $24,0 \pm 0,9$ & $661,3 \pm 42,1$ & 5,3 \\
1 & $25,4 \pm 4,3$ & $57,9 \pm 1,5$ & $11,2 \pm 0,8$ & 15,3 & $25,5 \pm 1,0$ & $646,0 \pm 40,3$ & 5,2 \\
2 & $23,9 \pm 4,7$ & $58,8 \pm 1,7$ & $11,6 \pm 0,9$ & 16,3 & $26,0 \pm 1,0$ & $623,6 \pm 48,6$ & 5,1 \\
3 & $22,2 \pm 5,5$ & $60,8 \pm 1,8$ & $12,3 \pm 0,5$ & 19,2 & $27,3 \pm 1,1$ & $607,0 \pm 42,3$ & 5,0 \\
4 & $21,3 \pm 4,0$ & $61,0 \pm 1,6$ & $12,6 \pm 0,3$ & 18,6 & $27,4 \pm 1,1$ & $583,3 \pm 50,1$ & 4,8 \\
5 & $16,0 \pm 4,1$ & $67,3 \pm 3,1$ & $13,0 \pm 0,9$ & 26,0 & $31,6 \pm 1,3$ & $506,5 \pm 46,3$ & 5,2 \\
6 & $18,9 \pm 3,6$ & $61,6 \pm 2,9$ & $12,8 \pm 0,5$ & 17,3 & $27,9 \pm 1,1$ & $527,1 \pm 42,1$ & 4,8 \\
7 & $22,6 \pm 4,9$ & $58,6 \pm 3,1$ & $12,4 \pm 0,6$ & 14,6 & $25,9 \pm 1,0$ & $586,1 \pm 40,5$ & 4,7 \\
\hline
\end{tabular}

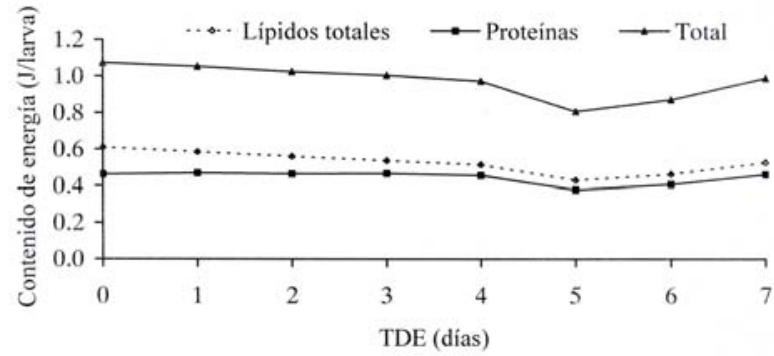

Figura 4

Cambios en los contenidos de carbono (C), nitrógeno (N) y cenizas durante el desarrollo larval de besugo

Variations in the carbon $(\mathrm{C})$, nitrogen $(\mathrm{N})$ and ash content during the larval development of red porgy

El análisis de regresión potencial linearizado a la relación entre el consumo de oxígeno y el peso de las larvas (Fig. 6), resultó ser significativo $\left(\mathrm{F}_{(1,14)}=66,106\right.$; error estándar de $\left.\mathrm{b}_{(\mathrm{Sb})}=0,111947, P<0,000\right)$. La prueba de isometría para contrastar la hipótesis Ho: $\mathrm{b}=$ 1 contra Hi: $b \neq 1(P=0,435813)$, confirmó un cambio isométrico en relación al peso, con un exponente de masa $=0,91$. La tasa metabólica peso-específica $\left(\mu \mathrm{IO}_{2}\right.$ $\mathrm{g}^{-1} \min ^{-1}$ ) mostró un patrón ontogénico bifásico (Fig. 7,

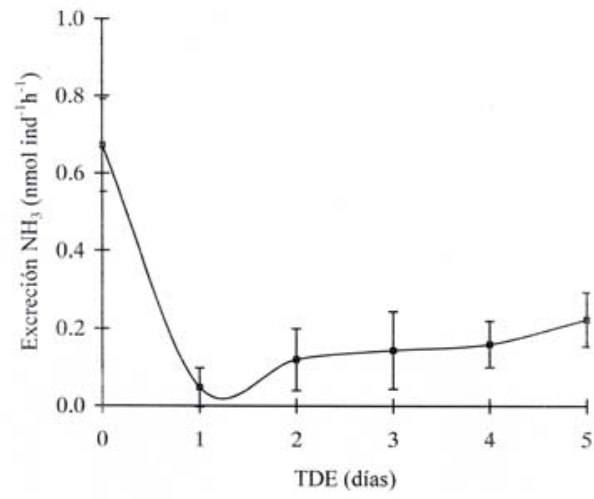

Figura 5

Excreción de amonio en larvas de besugo. Las barras verticales representan la desviación estándar

Ammonia excretion in red porgy larvae. Vertical bars represent the standard deviation

Tabla 3), que respondió al modelo $\mathrm{y}=\mathrm{a}+\mathrm{b} * \max \left(\mathrm{t}_{0}\right.$, $\mathrm{x})+\mathrm{c} * \min \left(\mathrm{t}_{0}, \mathrm{x}\right)$ : Fase $\mathrm{I}$, con un incremento lineal con la edad desde la eclosión hasta el día 6 aproximadamente, y Fase II, prácticamente constante con una suave pendiente negativa. En base a la relación entre el consumo de oxígeno y el peso seco, junto a los supuestos previamente mencionados, se pudo calcular el requerimiento alimenticio diario. 
Tabla 3

Resultados de la estimación estadística no lineal correspondiente a las curvas de la Fig. 7 (n=160; SE= error estándar)

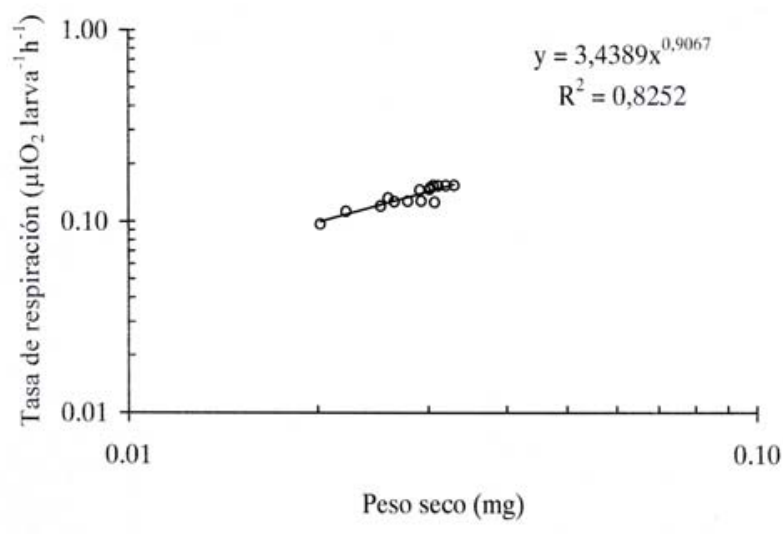

Figura 6

Relación entre la tasa de consumo de oxígeno y el peso seco en larvas de besugo

Relationship between oxygen consumption rate and dry weight in red porgy larvae

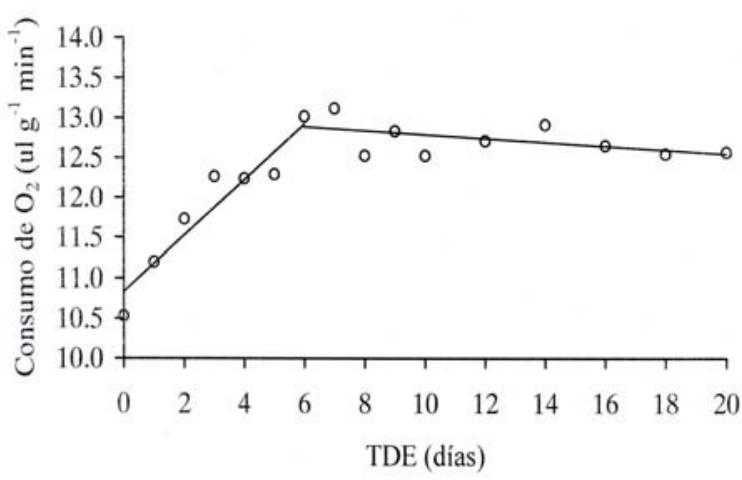

Figura 7

Cambios en el consumo de oxígeno peso-específico a lo largo del desarrollo de las larvas de besugo. Varianza explicada $=90,50 \%$

Changes in the relationship between mass-specific oxygen consumption during the development of red porgy larvae. Explained variance $=90.50 \%$
Non-lineal statistical parameters corresponding to Fig. 7 $(\mathrm{n}=160 ; \mathrm{SE}=$ standard error $)$

\begin{tabular}{lcccc}
\hline & $\mathrm{a}$ & $\mathrm{b}$ & $\mathrm{t} 0$ & $\mathrm{c}$ \\
\hline Parámetro & 10,96247 & $-0,02442$ & 5,819701 & 0,355899 \\
$\mathrm{SE}$ & 0,26541 & 0,01597 & 0,900489 & 0,083578 \\
$\mathrm{t}(12)$ & 41,30405 & $-1,52961$ & 6,462820 & 4,258287 \\
$P$ & 0,00000 & 0,15204 & 0,000031 & 0,001111 \\
\hline
\end{tabular}

\section{Discusión}

El besugo eclosiona de un huevo pelágico con la mayoría de sus órganos (boca, ano, branquias, ojos) en estado indiferenciado, los cuales se desarrollan rápidamente durante el final del periodo vitelino. Las mayores tasas de crecimiento en las larvas de besugo concuerdan con la rápida reducción de las reservas internas (saco vitelino y gota oleosa) y con el momento en que las larvas son capaces de consumir y utilizar alimento externo. Entre ambos periodos, se observó una fase de crecimiento lento asociada con altas mortalidades en el tanque de cría, observándose que, aunque la boca está abierta, las primeras partículas de alimento aparecen más tarde (día $4 \mathrm{DE}$ ), coincidiendo con la detección de actividad digestiva en el intestino medio (Roo et al. 1999). Estos cambios han sido documentados durante los mismos periodos larvales en otros espáridos como P. major (Fukuhara 1985), Acanthopagrus shlegeli (Fukuhara 1987), P. auratus (Pankhurst et al. 1991), Dentex gibbosus (FernándezPalacios et al. 1994) y D. dentex (Santamaría et al. 2004). El besugo exhibe un saco vitelino bastante pequeño, por lo que la larva debe comenzar a alimentarse ten rápido como sea posible. Este hecho se reflejó en el perfil del parámetro $\mathrm{Ed}$, el cual fue decreciendo y se redujo a cero simultáneamente con la finalización de las reservas de alimento, aumentando consecuentemente la mortalidad si la larva no consigue alimento.

El estadio de 4 días DE parece indicar el momento clave de desaparición del vitelo y la aparición de alimento externo en el tracto digestivo. Luego de la absorción de las reservas energéticas endógenas, las larvas de besugo comenzaron la ingestión de su primer 
alimento o empezaron a mostrar signos de ayuno, como por ejemplo, la contracción del intestino. La mayoría de las larvas de peces marinos comienzan su alimentación antes de que el saco vitelino se consuma completamente (Heming \& Buddington 1988). En nuestro estudio, el besugo completó la absorción del saco vitelino a los 3,316 $\pm 0,146 \mathrm{~mm} \mathrm{Lt}$ (4 días $\mathrm{DE})$, tiempo que coincidió con el inicio de la alimentación externa. Conides \& Glamuzina (2001) obtuvieron resultados similares para el besugo del Mar Mediterráneo. En P. major, la absorción del saco vitelino ocurre a los 3,2 mm Lt (Kitajima 1978).

En términos de tasa de consumo, se observaron dos fases distintivas en la absorción del vitelo a partir del momento de la eclosión. Una fase de post-eclosión, caracterizada por una tasa de absorción de vitelo relativamente alta y constante, y una segunda fase de absorción más lenta en la cual se terminaron de consumir las reservas internas, principalmente la gota oleosa. Existiría una tercera fase o pre-eclosión (PE), descripta por Heming \& Buddington (1988), caracterizada por un lento pero constante incremento de la tasa de absorción de vitelo dentro del huevo.

Los resultados revelan que la composición del cuerpo del besugo cambió básicamente durante la absorción del vitelo y luego del inicio de la alimentación exógena, como consecuencia de la transformación del vitelo en tejido larval o en energía. Luego de la eclosión y durante el estadio previo a la alimentación exógena, las larvas vitelinas consumieron preferentemente compuestos ricos en carbono. Los cambios en el contenido de $\mathrm{C}$ y $\mathrm{N}_{2}$ observados sugieren que durante el estadio vitelino, las larvas convierten preferentemente compuestos ricos en carbono (Tucker 1988), principalmente lípidos, y solo cuando las reservas de vitelo se acaban, lípidos y proteínas se consumen o incorporan a una tasa similar. Resultados coincidentes a los del presente trabajo fueron publicados para larvas de dorada S. aurata (Yúfera et al. 1993), de lenguado Pseudopleuronectes americanus (Cetta \& Capuzzo 1982) y de bacalao Gadus morhua (Jorgensen 1985).

Rønnestad et al. (1994) analizaron la composición relativa de aminoácidos (AA) y ácidos grasos (AG) en embriones y larvas vitelinas de $S$. aurata, y establecieron un "aminoácido promedio", cuya oxidación requiere 5,5 mol $\mathrm{O}_{2}$ y rinde 1,3 $\mathrm{mol} \mathrm{NH}_{3}$, y un "ácido graso promedio" unido a un triacilglicerol
(TGA), cuya oxidación requiere $77 \mathrm{~mol} \mathrm{O}_{2}$ por cada mol de TAG. Utilizando como supuesto el coeficiente de nitrógeno $\mathrm{NQ}=0,24$ (mol de $\mathrm{NH}_{3}$ producido / mol $\mathrm{O}_{2}$ consumido) derivado de estos cálculos para este espárido afín al besugo, podemos estimar la contribución de las proteínas y los ácidos grasos a la energía aeróbica del besugo (Tabla 4). La producción de amonio y su correspondiente NQ sugieren que el $49,63 \%$ de la energía disipada durante la eclosión fue aportada por las proteínas, pero solo un $12,39 \%$ durante la última parte del estadio vitelino (día $4 \mathrm{DE}$ ). La estrategia de utilización de estos compuestos durante los primeros días de vida de la larva de besugo indica que las proteínas del saco vitelino tienen una importante participación como combustible celular hasta el momento de la eclosión (Tabla 4). A partir de aquí, el decrecimiento de los valores de NQ con el desarrollo larval implica un cambio de proteínas a lípidos como principal sustrato energético. Los lípidos neutros, y en especial los triglicéridos (TAG), son considerados generalmente como las reservas de energía más importantes en los huevos de teleósteos marinos (Vetter et al. 1983, Blaxter 1988), siendo los aminoácidos libres y las proteínas una fuente endógena adicional (Fyhn 1989). El incremento en la tasa de absorción de la gota oleosa luego del día $1 \mathrm{DE}$, sugiere que los lípidos derivados de ella son el combustible aeróbico dominante para las larvas de besugo durante este breve estadio de desarrollo. A partir del día $5 \mathrm{DE}$, la hidrólisis proteica parece volver a incrementarse (Tabla 4), posiblemente debido al aporte de alimento externo que suministraría el $\mathrm{N}_{2}$ faltante para la producción de $\mathrm{NH}_{3}$, ya que el vitelo se ha consumido.

\section{Tabla 4}

\section{Cociente de nitrógeno (NQ) y porcentaje de disipación de energía aeróbica derivada del catabolismo de aminoácidos (AA) y ácidos grasos (AG) en larvas de besugo}

Nitrogen quotient (NQ) and percentage of aerobic energy dissipation from the catabolism of aminoacids (AA) and fatty acids (AG) in red porgy larvae

\begin{tabular}{cccc}
\hline TDE (días) & NQ & $\begin{array}{c}\text { \% energía } \\
\text { AA }\end{array}$ & $\begin{array}{c}\text { \% energía } \\
\text { AG }\end{array}$ \\
\hline 0 & 0,10 & 49,63 & 50,37 \\
1 & 0,01 & 3,50 & 96,50 \\
2 & 0,02 & 8,78 & 91,22 \\
3 & 0,02 & 10,59 & 89,41 \\
4 & 0,03 & 12,39 & 87,61 \\
5 & 0,05 & 21,56 & 78,44 \\
\hline
\end{tabular}


La transición de la alimentación endógena a exógena en las larvas de besugo, como ocurre en la mayoría de los teleósteos marinos, es un periodo de dramáticos cambios observados a nivel del contenido de lípidos y proteínas, del peso seco, del nivel de consumo de oxígeno y de los contenidos de $\mathrm{C} \mathrm{y} \mathrm{N}_{2}$.

\section{Tasa metabólica}

La tasa de respiración de los peces es directamente proporcional al peso del cuerpo elevado a la 0,80 (Winberg 1956, Paloheimo \& Dickie 1965). Brett \& Groves (1979) estimaron un valor medio para peces de $0,84 \pm 0,03$. Nuestro valor de $b=0,91$ se encuentra un poco por encima de lo establecido como general por estos autores, pero concuerda con los valores hallados por Glass (1969) en otras especies, quien encontró que el exponente del peso se ubicó entre $0,59-1,28$ con una media de 0,88 . Houde \& Scheckter (1983), trabajando con el espárido Archosargus rhomboidalis, estimaron una media de 0,88. Yoshinaga et al. (1994) e Imabayashi \& Takahashi (1987) obtuvieron valores superiores a los del presente trabajo, 0,92 y 0,98 respectivamente, para un rango más amplio de pesos larvales de P. major. Houde \& Scheckter (1983) obtuvieron altas constantes para las larvas de los peces subtropicales Anchoa mitchilli $(0,98)$ y Achirus lineatus $(0,94)$.

El consumo de oxígeno en larvas de besugo mostró un cambio isométrico en relación al peso $(\mathrm{b}=0,91)$, por lo que la tasa de consumo se considera prácticamente independiente del tamaño del cuerpo en este estadio del desarrollo. Resultados similares fueron encontrados en otras especies por Rombough (1988), Oikawa et al. (1991) y Oikawa \& Itazawa (1992).

En los primeros estadios de vida de los peces se observan diferentes patrones en la relación metabolismo-biomasa (Tabla 5). Durante el estadio vitelino, la tasa metabólica peso-específica se incrementó rápidamente en Cyclopterus lumpus (Davenport 1983) y en Tautoga onitis (Laurence 1973), mientras que se mantuvo prácticamente constante en Gadus morhua (Davenport \& Lönning 1980). En alevines de Salvelinus alpinus (Holeton 1973) y Coregonus sp. (Forstner et al. 1983), b se encontró cerca de la unidad (1,09 y 0,99 respectivamente). De alevines a juveniles de Oncorhynchus mykiss, b varió entre 0,93 y 0,96 (Weiser 1985).

Tabla 5

Análisis de regresión de las relaciones entre el consumo de oxígeno por unidad de masa $\mathrm{M} / \mathrm{W}\left(\mu \mathrm{lg}^{-1} \mathrm{~min}^{-1}\right)$ y la edad D

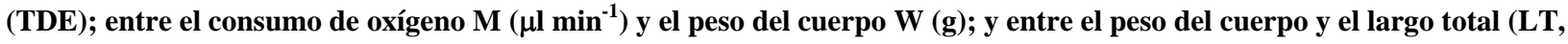
mm) en varias especies de peces

Regression analysis between oxygen consumption per mass unit $\mathrm{M} / \mathrm{W}\left(\mu \mathrm{g} \mathrm{g}^{-1} \mathrm{~min}^{-1}\right)$ and age $\mathrm{D}$ (TDE); between oxygen

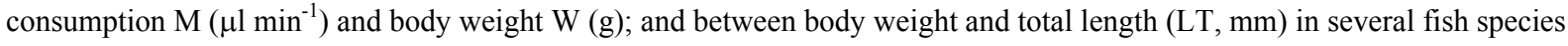

\begin{tabular}{|c|c|c|c|c|c|c|}
\hline \multirow[b]{2}{*}{ Especie } & \multirow[b]{2}{*}{ Estadio $^{1}$} & \multirow[b]{2}{*}{ Peso del cuerpo ${ }^{2}$} & \multirow{2}{*}{$\begin{array}{c}\text { Temp } \\
\left({ }^{\circ} \mathrm{C}\right)\end{array}$} & \multicolumn{2}{|c|}{ Constantes } & \multirow[b]{2}{*}{ Referencia } \\
\hline & & & & a & $\mathrm{b}$ & \\
\hline Cyclopterus lumpus & $\mathrm{V}$ & 0,76-0,86 (mgPS) & 5 & $*$ & $*$ & Davenport (1983) \\
\hline Tautoga onitis & $\mathrm{V}$ & $0,0015-0,0012(\mathrm{bPS})^{3}$ & 16 & $* *$ & $* *$ & Laurence (1973) \\
\hline Gadus morhua & $\mathrm{V}$ & $0,056(\mathrm{mgPS} \text { medio })^{3}$ & 5 & $* * *$ & $* * *$ & Davenport \& Lönning (1980) \\
\hline Salvelinus alpinus & $\mathrm{A}$ & $0,05-0,12(\mathrm{gPH})$ & 2 & 1,5 & 1,09 & Holeton (1973) \\
\hline Oncorhynchus mykiss & A-J & $0,08-7,0(\mathrm{gPH})$ & $4 ; 12$ & 1,$2 ; 2,5$ & 0,$96 ; 0,93$ & Weiser (1985) \\
\hline Coregonus sp. & V-P & $0,006-0,065(\mathrm{gPH})$ & 10 & 6,5 & 0,99 & Forstner et al. (1983) \\
\hline Anchoa mitchilli & $\mathrm{P}$ & 0,0089-0,42 (mgPS) & 26 & 93 & 0,98 & Houde \& Schekter (1983) \\
\hline Aechosargus rhomboidalis & $\mathrm{P}$ & $0,018-0,066(\mathrm{mgPS})$ & 26 & 32 & 0,84 & Houde \& Schekter (1983) \\
\hline Oreochromis niloticus & V-P & $0,00012-0,013(\mathrm{gPS})^{4}$ & 30 & 2,9 & 0,42 & DeSilva et al. (1986) \\
\hline \multicolumn{4}{|c|}{ 1. $\mathrm{V}=$ vitelino $; \mathrm{A}=$ alevino $\mathrm{J}=$ juvenil $; \mathrm{P}=$ post-larva } & \multicolumn{3}{|c|}{$*$ Consumo de oxígeno $=0,82-3,2 \mu \mathrm{mg}^{-1} \mathrm{~h}^{-1}$} \\
\hline \multicolumn{4}{|c|}{ 2. $\mathrm{PS}=$ peso seco; $\mathrm{PH}=$ peso húmedo } & \multicolumn{3}{|c|}{$* *$ Consumo de oxígeno $=3,7-9,1 \mu 1$ ind $^{-1}$ dia $^{-1}$} \\
\hline \multicolumn{4}{|c|}{ 3. Durante el estadio vitelino no se observaron cambios sustanciales } & \multirow{2}{*}{\multicolumn{3}{|c|}{$* * *$ Consumo de oxígeno $=1,6 \mu \mathrm{lmg}^{-1} \mathrm{~h}^{-1}$}} \\
\hline 4. Peso obtenido luego de extrac & r el vitelo & & & & & \\
\hline
\end{tabular}


En la relación bifásica observada entre el consumo de oxígeno de rutina y el estadio de desarrollo del besugo, la Fase I corresponde a un estadio de transición entre una larva vitelina hacia la post-larva carente de saco vitelino, la cual comenzaría a utilizar completamente su sistema branquial. El análisis estadístico dio como resultado un punto de inflexión correspondiente al día $6 \mathrm{DE}$, momento que coincidió con la aparición de la vejiga natatoria. A partir de aquí, la Fase II supone la utilización de branquias totalmente formadas y funcionales, con lo que la eficiencia de transferencia de oxígeno declinaría exponencialmente a medida que la relación superficie-volumen decrece durante esta fase de rápido crecimiento en volumen (Post \& Lee 1996). Este patrón bifásico del metabolismo de rutina observado en $P$. pagrus evidencia una alta tasa de consumo de oxígeno por unidad de peso en los primeros días de vida de la larva, para luego decrecer después de la apertura de la boca y la formación de las branquias. El intercambio de gases respiratorios es básicamente cutáneo hasta la apertura de la boca, por lo que el desarrollo completo del sistema respiratorio branquial debe estar listo para cuando la larva alcanza un cierto tamaño crítico.

\section{Conclusiones}

Las características del desarrollo larval del besugo muestran los importantes cambios que ocurren durante los primeros días de vida, y son una importante herramienta para ajustar las técnicas de producción de juveniles en cautiverio. El besugo debe hacer frente a una importante organogénesis durante los primeros tres días después de la eclosión, para lo cual cuenta con las reservas energéticas de un saco vitelino relativamente pequeño. El cuarto día de vida de estas larvas marca un momento crítico para la supervivencia. Es el momento de una prácticamente nula superposición entre alimentación endógena y exógena, por lo que la larva debe comenzar a alimentarse tan rápido como sea posible, y es donde deben volcarse los mayores esfuerzos tendientes a reducir los niveles de mortalidad en cautiverio de esta especie.

\section{Agradecimientos}

Al Lic. Daniel Hernández por el apoyo estadístico para el análisis de los datos y al Lic. Jorge Sánchez por la realización de los análisis químicos proximales. A los evaluadores anónimos de este trabajo por sus valiosas observaciones y sugerencias al mismo. Contribución INIDEP N $^{\circ} 1429$.

\section{Literatura citada}

Antonacopoulos N. 1968. Entwurf zum. FAOGeberalstandard CODEX Fish Al Anlage 3, pp. 11-12, Analysenmethoden.

Aristizabal EO. En prensa. Desove en cautiverio y calidad de los huevos de besugo, Pagrus pagrus. Serie INIDEP Informe Técnico 59: 12-17.

Aristizabal EO, MI Müller, GA Bambill, AV López, M Sabatini, M Costagliola, S Incorvaia, A Vega, JC Carrizo \& E Manca. 1997a. Producción de alimento vivo y cría de besugo, período 1995-1996. Informe Técnico Interno DNI-INIDEP 83, 92 pp.

Aristizabal EO, MI Müller, AV López, GA Bambill \& M Sabatini. 1997b. Producción de microalgas y rotíferos y su aplicación en piscicultura marina. Informe Técnico Interno DNI-INIDEP 59, 16 pp.

Brett JR \& TDD Groves. 1979. Physiological Energetics. En: Hoar WS, DJ Randall \& JR Brett (eds), Fish Physiology 8: 279-252, Academic Press, Nueva York.

Blaxter JHS. 1988. Patterns and variety in development. En: Hoar WS \& DJ Randall (eds), Fish Physiology 11A: 1-58, Academic Press, Nueva York.

Cech JJ. 1990. Respirometry. En: Schreck CB \& PB Moyle (eds), Methods for fish biology, pp. 335-356, American Fisheries Society, USA.

Cetta CM \& JM Capuzzo. 1982. Physiological and biochemical aspects of embryonic and larval development of the winter flounder, Pseudopleuronectes americanus. Marine Biology 71: 327-337.

Conides AJ \& B Glamuzina. 2001. Study on the early larval development and growth of the red porgy, Pagrus pagrus with emphasis on the mass mortalities observed during this phase. Scientia Marina 65(3): 193-200.

Cotrina CP. 1989. Estudio biológico del besugo (Pagrus pagrus) del Ecosistema Costero Bonaerense. Tesis Doctoral, Universidad de Buenos Aires, $161 \mathrm{pp}$.

Davenport J. 1983. Oxygen and the developing eggs and larvae of the lumpfish, Cyclopterus lumpus. Journal of the Marine Biological Association of the United Kingdom 63: 633-640

Davenport J \& S Lönning. 1980. Oxygen uptake in developing eggs and larvae of the cod, Gadus morhua L. Journal of Fish Biology 16: 249-256. 
El-Rayis O. 1985. Re-assessment of the titration method for determination of organic carbon in recent sediments. Rapport de la Commission Internationale de la Mer Méditerranee 29(7): 45-47.

Fernández-Palacios H, D Montero, J Socorro, MS Izquierdo \& JM Vergara. 1994. First studies on spawning, embryonic and larval development of Dentex gibbosus (Rafinesque, 1810) (Osteichthyes, Sparidae) under controlled conditions. Aquaculture 122: 63-73.

Forstner H, S Hinterleitner, K Mähr \& W Weiser. 1983. Towards a better definition of "metamorphosis" in Coregonus sp.: Biochemical, histological, and physiological data. Canadian Journal of Fisheries and Aquatic Sciences 40: 1224-1232.

Fukuhara O. 1985. Functional morphology and behaviour of early life stages of red sea bream. Bulletin of the Japanese Society of Scientific Fisheries 51: 731-743.

Fukuhara O. 1987. Larval development and behaviour in early life stages of black sea bream reared in the laboratory. Bulletin of the Japanese Society of Scientific Fisheries 53: 371-379.

Fyhn HJ. 1989. First feeding of marine fish larvae. Are free amino acids the source of energy? Aquaculture 88: 111120 .

Gaudette HE, WR Flight, L Toner \& W Folger. 1974. An inexpensive titration method for the determination of organic carbon in recent sediments. Journal of Sedimentary Petrology 44 (1): 249-253.

Glass NR. 1969. Discussion of calculation of power function with special reference to respiratory metabolism in fish. Journal of the Fisheries Research Board of Canada 26: 2643-2650.

Guinea J \& F Fernandez. 1997. Effect of feeding frequency, feeding level and temperature on energy metabolism in Sparus aurata. Aquaculture 148: 125-142.

Heming TA \& RK Buddington. 1988. Yolk absorption in embryonic and larval fishes. En: Hoar WS \& DJ Randall (eds), Fish Physiology 11: 407-446, Academic Press, Nueva York.

Hernández-Cruz CM, M Salhi, M Bessonart, MS Izquierdo, MM González \& H Fernández-Palacios. 1999. Rearing techniques for red porgy (Pagrus pagrus) during larval development. Aquaculture 179: 489-497.

Holeton GF. 1973. Respiration of the arctic charr (Salvelinus alpinus) from a high arctic lake. Journal of the Fisheries Research Board of Canada 30: 717-723.
Houde ED \& RC Scheckter. 1983. Oxygen uptake and comparative energetics among eggs and larvae of therr subtropical marine fishes. Marine Biology 72: 283-293.

Imabayashi H \& M Takahashi. 1987. Oxygen consumption of postlarval and juvenile red sea bream, Pagrus major, with special reference to group effect. Journal of the Faculty of Applied Biological Sciences of Hiroshima University 26: 15-21.

Ishibashi Y, K Kato, S Ikeda, O Murata, T Nasu \& H Kumai. 1992. Effect of dietary ascorbic acid on the tolerance for intermittent hypoxic stress in Japanese parrot fish. Nippon Suisan Gakkaishi 58: 2147-2152.

Jorgensen L. 1985. Carbon and nitrogen utilization in developing eggs and larvae of cod (Gadus morhua, L) and variation among different parents. Fishery Research 3: $337-342$.

Kamler E. 1992. Early life history of fish, 183 pp, Chapman \& Hall, Londres.

Kitajima C. 1978. Acquisition of fertilized eggs and massculture of juveniles of red seabream, Pagrus major. Special Repport of the Nagasaki Prefecture Institute of Fisheries 5, 92 pp. (En japonés).

Klaoudatos S, N Tsevis \& A Conides. 1990. Energy sources during early larval development of the European sea bass, Dicentrarchus labrax (L). Aquaculture 87: 361-372.

Laurence GC. 1973. Influence of temperature on energy utilization of embryonic and prolarval tautog, Tautoga onitis. Journal of the Fisheries Research Board of Canada 30: $435-442$.

Manooch III CS. 1976. Reproductive cycle, fecundity and sex ratio of the red porgy Pagrus pagrus (Pisces: Sparidae) in North Carolina. Fisheries Bulletin 74: 775781.

Mihelakakis A, T Yoshimatsu \& C Tsolkas. 2001. Spawning in captivity and early life history of cultured red porgy, Pagrus pagrus. Aquaculture 199: 333-352.

Mylonas CC, M Papadaki, M Pavlidis \& $\mathbf{P}$ Divanach. 2004. Evaluation of egg production and quality in the Mediterranean red porgy (Pagrus pagrus) during two consecutive spawning seasons. Aquaculture 232: 637649.

Oikawa S \& Y Itazawa. 1992. Comparision of oxygen consumption of larval and juvenile carp determined by three different methods. Fishery Sciences 61: 487-490.

Oikawa S, Y Itazawa \& M Gotoh. 1991. Ontogenetic changes in the relationship between metabolic rate and body mass in a sea bream Pagrus major (Temminck \& Schlegel). Journal of Fish Biology 38(4): 483-496. 
Pankhurst PM, JC Montgomery \& NW Pankhurst. 1991. Growth, development and behaviour of artificially reared larval Pagrus auratus (Block and Schneider, 1801) (Sparidae). Australian Journal of Marine and Freshwater Research 42: 391-398.

Paloheimo JE \& LM Dickie. 1965. Food and growth of fishes. II. Effects of food and temperature on the relation between metabolism and body weight. Journal of the Fisheries Research Board of Canada 23(6): 869-908.

Phil L, SP Baden, RJ Díaz \& LC Schaffner. 1992. Hypoxia-induced structural changes in the diet of bottomfeeding fish and Crustacea. Marine Biology 112: 349361.

Post JR \& JA Lee. 1996. Metabolic ontogeny of teleost fishes. Canadian Journal of Fisheries and Aquatic Sciences 53: 910-923.

Radonic M, AV López, M Oka \& EO Aristizabal. 2005. Effect of the incubation temperature on the embryonic development and hatching time of eggs of the red porgy Pagrus pagrus (Linné, 1758) (Pisces: Sparidae). Revista de Biología Marina y Oceanografía 40(2): 91-99.

Ricker WE. 1979. Growth rates and models. In: Hoar WS \& DJ Randall (eds.). Fish Physiology, VIII Bioenergetics and Growth, pp. 677-743, Academic Press, Nueva York.

Rombough PJ. 1988. Respiration gas exchange, aerobic metabolism, and effect of hypoxia during early life. En: Hoar WS \& DJ Randall (eds), Fish Physiology 2A: 59161, Academic Press, Nueva York.

Rønnestad I, WM Koven, A Tandler, M Harel \& HJ Fyhn. 1994. Energy metabolism during development of eggs and larvae of gilthead seabream (Sparus aurata). Marine Biology 120: 187-196.

Roo FJ, J Socorro, MS Izquierdo, MJ Caballero, CM Hernández-Cruz, A Fernández \& H FernándezPalacios. 1999. Development of red porgy Pagrus pagrus visual system in relation with changes in the digestive tract and larval feeding habits. Aquaculture 179: 499-512.

SAGPyA. 2004. Exportación de productos pesqueros. Secretaría de Agricultura, Ganadería, Pesca y
Alimentación, Dirección Nacional de Pesca y Acuicultura, 9 pp., Buenos Aires, Argentina.

Santamaría CA, M Marín de Mateoa, $\mathbf{R}$ Traveseta, $\mathbf{R}$ Salab, A Grauc, E Pastore, C Sarasqueted \& S Crespo. 2004. Larval organogenesis in common dentex Dentex dentex L. (Sparidae): histological and histochemical aspects. Aquaculture 237: 207-228.

Tanaka M. 1985. Factors affecting the insore migration of pelagic larval and demersal juvenile red sea bream Pagrus major to a nursery ground. Transactions of the American Fisheries Society 114: 471-477.

Tucker Jr JW. 1988. Energy utilization in bay anchovy, Anchoa mitchilli, and black sea bass, Centropristris striata striata, egg and larvae. Fishery Bulletin 78: 279293.

Vetter RD, RE Hudson \& C Arnold. 1983. Energy metabolism in a rapidly developing marine fish egg, the red drum (Sciaenops ocellata). Canadian Journal of Fisheries and Aquatic Sciences 40: 627-634.

Weiser W. 1985. Developmental and metabolic constraints of the scope for activity in young rainbow trout (Salmo gairdneri). Journal of Experimental Biology 118: 133142.

Winberg GG. 1956. Rate of metabolism and food requirements of fishes. Journal of Fisheries Research Board of Canada, Translation Series 194, 202 pp.

Wu RSS. 2002. Hypoxia: from molecular response to ecosystem responses. Marine Pollution Bulletin 45: 3545 .

Yoshinaga K, J Hiromi \& S Kadota. 1994. Respiration and food requirement by larvae and juveniles of red seabream, Pagrus major. Bulletin of the College of Agriculture Veterinary Medicine of the Nihon University 51: 174181.

Yúfera M, A Polo \& E Pascual. 1993. Changes in chemical composition and biomass during the transition from endogenous to exogenous feeding of Sparus aurata L. (Pisces, Sparidae) larvae reared in the laboratory. Journal of Experimental Marine Biology and Ecology 167: 149161. 